\title{
ON THE ASYMPTOTIC SOLUTION OF THE LAGERSTROM MODEL EQUATION*
}

\author{
S. ROSENBLAT AND J. SHEPHERD $\dagger$
}

\begin{abstract}
The Lagerstrom equation is a one-dimensional model of the equations of viscous flow at low Reynolds numbers. It is shown how a uniformly valid asymptotic solution to the Lagerstrom equation can be obtained by an iteration procedure applied directly to an equivalent integral equation, and without recourse to inner and outer expansions.
\end{abstract}

1. Introduction. We shall be concerned in this paper with finding an asymptotic approximation as $\varepsilon \rightarrow 0$ to the solution $u(r, \varepsilon)$ of the equation

$$
u^{\prime \prime}+\frac{k}{r} u^{\prime}+\varepsilon u u^{\prime}=0
$$

$1<r<\infty, 0<\varepsilon \leqq \varepsilon_{0}, k$ a positive integer, subject to the boundary conditions

$$
u(1, \varepsilon)=0, \quad u(\infty, \varepsilon)=1 .
$$

The system (1.1)-(1.2), henceforth referred to as Problem A, has been proposed by Lagerstrom [5] as a simple model to describe, for sufficiently small $\varepsilon$, the characteristics of low Reynolds number flow past an obstacle. (Here $\varepsilon$ represents the Reynolds number; $k=1$ corresponds to the flow past a circular cylinder and $k=2$ to the flow past a sphere.) Moreover, Problem A is a good example of a singular perturbation problem, since the solution obtained by setting $\varepsilon=0$ in $(1.1)-(1.2)$ is not a uniformly valid approximation on $1 \leqq r<\infty$ to the solution of Problem A. For this reason a number of authors (Lagerstrom [5], Cole [3], Bush [2], Hsiao [4]) have used Problem A to illustrate the construction of inner and outer expansions for small $\varepsilon$, and the application of matching methods.

Lagerstrom [5] and Cole [3] have suggested that a uniformly valid first approximation as $\varepsilon \rightarrow 0$ to the solution of Problem $\mathrm{A}$ is the solution $U(r, \varepsilon)$ of the equation

$$
U^{\prime \prime}+(k / r) U^{\prime}+\varepsilon U^{\prime}=0
$$

$1<r<\infty$, with the boundary conditions

$$
U(1, \varepsilon)=0, \quad U(\infty, \varepsilon)=1 .
$$

The system (1.3)-(1.4) will be called Problem B. It is a model of the Oseen equation for low Reynolds number flow.

Our primary objectives in this paper are

(i) to show that the solution to Problem B is, in an appropriate sense, an asymptotic approximation as $\varepsilon \rightarrow 0$ to the solution of Problem A;

(ii) to show that the solution of Problem $\mathrm{A}$ has a generalized asymptotic expansion (van der Corput [7]) as $\varepsilon \rightarrow 0$; that is, there exists an asymptotic

* Received by the editors March 18, 1974, and in revised form June 24, 1974.

$\uparrow$ Department of Mathematics, University of Melbourne, Victoria 3052, Australia. 
sequence $\left\{v_{i}(\varepsilon)\right\}_{i=0}^{\infty}$ and a sequence of functions $\left\{u_{i}(r, \varepsilon)\right\}_{i=0}^{\infty}$ such that

$$
u(r, \varepsilon)=\sum_{i=0}^{N} v_{i}(\varepsilon) U_{i}(r, \varepsilon)+O\left(v_{N+1}(\varepsilon)\right),
$$

$N=0,1,2, \cdots$, uniformly on $1 \leqq r<\infty$, with, in particular,

$$
v_{0}(\varepsilon)=1 \quad \text { and } \quad u_{0}(r, \varepsilon)=U(r, \varepsilon)
$$

(iii) to provide an algorithm for constructing the functions $u_{i}(r, \varepsilon)$.

In the course of establishing these results we shall also prove the existence and uniqueness of a solution to Problem A for sufficiently small $\varepsilon$.

Because of its physical interpretation, the case $k=1$ is of particular interest. This case has been studied exhaustively by Hsiao [4], who has proved the existence of two asymptotic expansions of Poincare type, which are the inner and outer expansions of the solution $u(r, \varepsilon)$.

The methods adopted in this paper make no use of inner and outer expansions, or of matching principles. Our aim is to go directly to the "composite" expansion in the form (1.5). Thus our work can be regarded as complementary to that of the authors cited above.

In $\S 2$ we develop some a priori estimates for the solution of Problem A, which then enable us, in $\S 3$, to prove that the solution exists and is unique. The results on asymptoticity are then given in $\S 4$.

2. A priori estimates. We begin by observing that for each $\varepsilon>0$ Problem $\mathrm{B}$ has a unique solution $U(r, \varepsilon)$ which may be written in the form

$$
U(r, \varepsilon)=1-W(r, \varepsilon),
$$

where

$$
W(r, \varepsilon)=\Gamma_{k}(\varepsilon r) / \Gamma_{k}(\varepsilon)
$$

and

$$
\Gamma_{k}(\varepsilon r)=\varepsilon^{1-k} \int_{r}^{\infty} s^{-k} e^{-\varepsilon s} d s=\int_{\varepsilon r}^{\infty} s^{-k} e^{-s} d s .
$$

$\Gamma_{k}$ is an incomplete gamma function and is related to the standard exponential integral

$$
E_{k}(x)=\int_{1}^{\infty} s^{-k} e^{-x s} d s
$$

([1, p. 228]) by the formula

$$
\Gamma_{k}(x)=x^{1-k} E_{k}(x)
$$

From (2.2), (2.3) and (2.5) we have that

$$
W^{\prime}(r, \varepsilon)=-r^{-k} e^{-\varepsilon r} / E_{k}(\varepsilon),
$$

a result which will be useful later. 
It follows immediately from (2.1)-(2.3) and (2.6) that

$$
0<W(r, \varepsilon) \leqq 1, \quad 0 \leqq U(r, \varepsilon)<1,
$$

and

$$
W^{\prime}(r, \varepsilon)<0, \quad U^{\prime}(r, \varepsilon)>0
$$

on $1 \leqq r<\infty, \varepsilon>0$.

Reverting now to Problem A, we define

$$
w=w(r, \varepsilon) \equiv 1-u(r, \varepsilon) .
$$

Integration of (1.1) then gives

$$
w^{\prime}(r, \varepsilon)=-C e^{\varepsilon} r^{-k} e^{-\varepsilon r} \cdot \exp \left(\varepsilon \int_{1}^{r} w d s\right),
$$

where

$$
C=C(\varepsilon)=-w^{\prime}(1, \varepsilon) .
$$

Integrating (2.10) and applying the boundary conditions (1.2) we obtain

$$
w(r, \varepsilon)=C e^{\varepsilon} \int_{r}^{\infty} t^{-k} e^{-\varepsilon t} \cdot \exp \left(\varepsilon \int_{1}^{t} w d s\right) d t
$$

and

$$
C e^{\varepsilon} \int_{1}^{\infty} t^{-k} e^{-\varepsilon t} \cdot \exp \left(\varepsilon \int_{1}^{t} w d s\right) d t=1 .
$$

From (2.9)-(2.13) it is evident that

$$
0<w(r, \varepsilon) \leqq 1, \quad 0 \leqq u(r, \varepsilon)<1
$$

and

$$
w^{\prime}(r, \varepsilon)<0, \quad u^{\prime}(r, \varepsilon)>0,
$$

$1 \leqq r<\infty, \varepsilon>0$, for every solution of Problem A.

The bounds (2.14) and (2.15) are not sharp enough for our subsequent purposes. We proceed to derive some sharper estimates for the solutions of Problem A.

We introduce functions $\alpha(r, \varepsilon)$ and $\beta(r, \varepsilon)$ defined by the formulas

$$
w(r, \varepsilon)=\alpha(r, \varepsilon) W(r, \varepsilon), \quad w^{\prime}(r, \varepsilon)=\beta(r, \varepsilon) W^{\prime}(r, \varepsilon) .
$$

Substituting for $W$ from (2.2)-(2.5) and for $w$ from (2.12) we easily see that

$$
\alpha(r, \varepsilon)=\frac{C e^{\varepsilon} E_{k}(\varepsilon) \int_{r}^{\infty} t^{-k} e^{-\varepsilon t} \cdot \exp \left(\varepsilon \int_{1}^{t} w d s\right) d t}{\int_{r}^{\infty} t^{-k} e^{-\varepsilon t} d t} .
$$

Now if we use the bound $w>0$ in (2.13) we infer at once, with the aid of (2.4), that

$$
0 \leqq C e^{\varepsilon} E_{k}(\varepsilon) \leqq 1
$$


Applying this to (2.17) we obtain

$$
0 \leqq \alpha(r, \varepsilon) \leqq e^{\varepsilon \mu},
$$

where

$$
\mu=\mu(\varepsilon) \equiv \int_{1}^{\infty} w(s, \varepsilon) d s .
$$

Similarly we find from (2.6), (2.10) and (2.18) that

$$
0 \leqq \beta(r, \varepsilon) \leqq e^{\varepsilon \mu} .
$$

It should be emphasized that both (2.19) and (2.21) hold uniformly on $1 \leqq r<\infty$.

We shall now show that $e^{\varepsilon \mu}=O(1)$ as $\varepsilon \rightarrow 0$. Let $r_{0}=r_{0}(\varepsilon)$ be the value of $r$ at which $w(r, \varepsilon)=\frac{1}{2}$. Since $w$ is a monotonically decreasing function of $r$, it is clear that

$$
w(r, \varepsilon)<\frac{1}{2} \quad \text { when } r>r_{0} .
$$

It follows from (2.12) that when $r \geqq r_{0}$,

$$
\begin{aligned}
w(r, \varepsilon) & =C e^{\varepsilon} \int_{r}^{\infty} t^{-k} e^{-\varepsilon t} \cdot \exp \left(\varepsilon \int_{1}^{r_{0}} w d s\right) \cdot \exp \left(\varepsilon \int_{r_{0}}^{t} w d s\right) d t \\
& \leqq C e^{(1 / 2) \varepsilon r_{0}} \int_{r}^{\infty} t^{-k} e^{-(1 / 2) \varepsilon t} d t .
\end{aligned}
$$

Thus we have

$$
\begin{aligned}
\mu & =\int_{1}^{\infty} w(r, \varepsilon) d r=\int_{1}^{r_{0}} w(r, \varepsilon) d r+\int_{r_{0}}^{\infty} w(r, \varepsilon) d r \\
& \leqq\left(r_{0}-1\right)+C e^{(1 / 2) \varepsilon r_{0}}\left(\frac{\varepsilon}{2}\right)^{k-2}\left\{\Gamma_{k-1}\left(\frac{1}{2} \varepsilon r_{0}\right)-\frac{1}{2} \varepsilon r_{0} \Gamma_{k}\left(\frac{1}{2} \varepsilon r_{0}\right)\right\}
\end{aligned}
$$

where we have substituted from (2.23) and reversed the order of the integrations.

To find an actual bound for $\mu$ we need to estimate $r_{0}$. When $r=r_{0}$ the inequality (2.23) becomes

$$
\frac{1}{2} \leqq \frac{e^{-\varepsilon+(1 / 2) \varepsilon r_{0}}}{E_{k}(\varepsilon)} \int_{r_{0}}^{\infty} t^{-k} e^{-(1 / 2) \varepsilon t} d t,
$$

where (2.18) has been used to replace $C$. With the aid of (2.3) and (2.5), (2.25) takes the form

$$
2^{k-2} e^{\varepsilon} \Gamma_{k}(\varepsilon) \leqq e^{(1 / 2) \varepsilon r_{0}} \Gamma_{k}\left(\frac{1}{2} \varepsilon r_{0}\right)
$$

Standard results on exponential integrals $([1$, p. 229]) give that, as $x \rightarrow 0$,

$$
\begin{gathered}
E_{0}(x) \sim 1 / x, \quad E_{1}(x) \sim \log (1 / x), \\
E_{k}(x) \sim 1 /(k-1) \quad \text { when } k \geqq 2 .
\end{gathered}
$$


Hence by (2.5) the analogous results as $x \rightarrow 0$ for the $\Gamma_{k}$ are

$$
\begin{gathered}
\Gamma_{0}(x) \sim 1, \quad \Gamma_{1}(x) \sim \log (1 / x), \\
\Gamma_{k}(x) \sim x^{1-k} /(k-1) \quad \text { when } k \geqq 2 .
\end{gathered}
$$

These show that the left-hand side of (2.26) becomes unbounded as $\varepsilon \rightarrow 0$. Therefore, in order that (2.26) be satisfied, we must have, as $\varepsilon \rightarrow 0$,

$$
\begin{gathered}
r_{0}=O(1) \quad \text { when } k \geqq 2, \\
\varepsilon r_{0}=o(1) \quad \text { when } k=1 .
\end{gathered}
$$

Applying (2.28) and (2.29) to (2.24) we find at once that

$$
\varepsilon \mu=O(1) \quad \text { as } \varepsilon \rightarrow 0, \quad k=1,2, \cdots .
$$

Hence it follows that $e^{\varepsilon \mu}=O(1)$ as $\varepsilon \rightarrow 0$, which we set out to prove.

We have thus established the following result.

Lemma 1. Every solution of Problem A can be represented in the form (2.16), with

$$
\alpha(r, \varepsilon)=O(1), \quad \beta(r, \varepsilon)=O(1)
$$

as $\varepsilon \rightarrow 0$, uniformly on $1 \leqq r<\infty$.

3. Existence and uniqueness. We shall transform Problem $A$ into a pair of integral equations, which will be the context for our subsequent discussion of the existence, uniqueness and asymptotic behavior of the solution.

We write (1.1) in the form

$$
u^{\prime \prime}+(k / r) u^{\prime}+\varepsilon u^{\prime}=\varepsilon(1-u) u^{\prime} .
$$

By introducing the appropriate Green's function for the operator on the lefthand side of (3.1), we can convert Problem A into an integral equation. After some straightforward algebraic manipulation we obtain

$$
w(r, \varepsilon)=W(r, \varepsilon)+\varepsilon \int_{1}^{\infty} K(s, r, \varepsilon) w(s, \varepsilon) w^{\prime}(s, \varepsilon) d s,
$$

where

$$
K(s, r, \varepsilon)= \begin{cases}\frac{(1-W(s, \varepsilon)) W(r, \varepsilon)}{W^{\prime}(s, \varepsilon)}, & 1 \leqq s \leqq r, \\ \frac{W(s, \varepsilon)(1-W(r, \varepsilon))}{W^{\prime}(s, \varepsilon)}, & r \leqq s<\infty,\end{cases}
$$

and where $w$ is defined in (2.9). Differentiation of (3.2) gives a second equation, namely

$$
w^{\prime}(r, \varepsilon)=W^{\prime}(r, \varepsilon)+\varepsilon \int_{1}^{\infty} \frac{\partial K}{\partial r}(s, r, \varepsilon) w(s, \varepsilon) w^{\prime}(s, \varepsilon) d s
$$

Equations (3.2) and (3.4) are conveniently transformed into a pair of integral equations for the functions $\alpha$ and $\beta$ defined in formulas (2.16). On performing 
the requisite substitutions we obtain

$$
\begin{aligned}
& \alpha(r, \varepsilon)=1+\varepsilon \int_{1}^{\infty} A(s, r, \varepsilon) \alpha(s, \varepsilon) \beta(s, \varepsilon) d s, \\
& \beta(r, \varepsilon)=1+\varepsilon \int_{1}^{\infty} B(s, r, \varepsilon) \alpha(s, \varepsilon) \beta(s, \varepsilon) d s,
\end{aligned}
$$

where

$$
A(s, r, \varepsilon)= \begin{cases}W(s, \varepsilon)(1-W(s, \varepsilon)), & 1 \leqq s \leqq r, \\ \frac{W^{2}(s, \varepsilon)(1-W(r, \varepsilon))}{W(r, \varepsilon)}, & r \leqq s<\infty,\end{cases}
$$

and

$$
B(s, r, \varepsilon)= \begin{cases}W(s, \varepsilon)(1-W(s, \varepsilon)), & 1 \leqq s \leqq r \\ -W^{2}(s, \varepsilon), & r \leqq s<\infty\end{cases}
$$

We thus have the following lemma.

Lemma 2. For fixed $\varepsilon>0$ every solution of Problem $\mathrm{A}$ is expressible in the form (2.16), where $\alpha, \beta$ are solutions of the integral equations (3.5) and (3.6).

The converse of this is the following.

LEMMA 3. If $(\alpha, \beta)$ is a solution of (3.5) and (3.6) which is bounded and continuous on $1 \leqq r<\infty$ for fixed $\varepsilon>0$, and which is such that $\alpha=O(1), \beta=O(1)$ as $\varepsilon \rightarrow 0$ uniformly on $1 \leqq r<\infty$, then the function $u(r, \varepsilon)$ defined by (2.9) and (2.16) is a solution to Problem A.

This can be verified by direct substitution.

We shall use contraction mapping to prove that a solution to (3.5)-(3.6) exists and is unique. To do this we first determine a bound for the kernels of these equations.

LEMMA 4. There exists an $h_{k}=h_{k}(\varepsilon)$ such that

$$
\varepsilon \int_{1}^{\infty}|A(s, r, \varepsilon)| d s \leqq h_{k}
$$

and

$$
\varepsilon \int_{1}^{\infty}|B(s, r, \varepsilon)| d s \leqq h_{k}
$$

for all $1 \leqq r<\infty$ and all $\varepsilon>0$. Moreover, as $\varepsilon \rightarrow 0$,

$$
h_{1}=O\left(\frac{1}{\log (1 / \varepsilon)}\right) ; \quad h_{2}=O\left(\varepsilon \log \frac{1}{\varepsilon}\right) ; \quad h_{k}=O(\varepsilon), \quad k \geqq 3 .
$$


Proof. From (3.7) and (2.7) we have

$$
\begin{aligned}
\varepsilon \int_{1}^{\infty}|A(s, r, \varepsilon)| d s= & \varepsilon \int_{1}^{r} W(s, \varepsilon)(1-W(s, \varepsilon)) d s \\
& +\varepsilon \frac{1-W(r, \varepsilon)}{W(r, \varepsilon)} \int_{r}^{\infty} W^{2}(s, \varepsilon) d s \\
\leqq & \varepsilon \int_{1}^{\infty} W(s, \varepsilon) d s
\end{aligned}
$$

Similarly, from (3.8),

$$
\begin{aligned}
\varepsilon \int_{1}^{\infty}|B(s, r, \varepsilon)| d s & \leqq \varepsilon \int_{1}^{r} W(s, \varepsilon)(1-W(s, \varepsilon)) d s+\varepsilon \int_{r}^{\infty} W^{2}(s, \varepsilon) d s \\
& \leqq \varepsilon \int_{1}^{\infty} W(s, \varepsilon) d s
\end{aligned}
$$

Now (2.2) gives

$$
\varepsilon \int_{1}^{\infty} W(s, \varepsilon) d s=\frac{\varepsilon}{\Gamma_{k}(\varepsilon)} \int_{1}^{\infty} \Gamma_{k}(\varepsilon s) d s=\varepsilon\left\{\frac{E_{k-1}(\varepsilon)}{E_{k}(\varepsilon)}-1\right\},
$$

on performing the integration and using (2.5). If now we define

$$
h_{k}(\varepsilon) \equiv \varepsilon\left\{\frac{E_{k-1}(\varepsilon)}{E_{k}(\varepsilon)}-1\right\} \text {, }
$$

then the first part of the Lemma is proved. The second part, that is, (3.11), follows immediately from the asymptotic estimates (2.27).

The next step is to define a suitable space in which contraction mapping can be applied.

Let $\Omega$ be the set

$$
\Omega=\left\{(r, \varepsilon): 1 \leqq r<\infty, 0<\varepsilon \leqq \varepsilon_{0}\right\},
$$

where $\varepsilon_{0}$ is sufficiently small that the estimates $(3.11)$ hold. Let $C_{M}(\Omega)$ be the set of ordered pairs of functions $(\alpha(r, \varepsilon), \beta(r, \varepsilon))$ which are continuous on $\Omega$ and are uniformly bounded in the sense that

$$
|\alpha(r, \varepsilon)| \leqq M, \quad|\beta(r, \varepsilon)| \leqq M \quad \forall(r, \varepsilon) \in \Omega,
$$

where $M$ is a constant (which may assume different values for different values of $k$ ). For brevity we denote by $\xi$ the elements of $C_{M}(\Omega)$ :

$$
\xi=(\alpha, \beta) \text {. }
$$

For each $\varepsilon \in\left(0, \varepsilon_{0}\right]$ we define the function

$$
\rho_{\varepsilon}\left(\xi_{1}, \xi_{2}\right)=\max \left\{\sup _{1 \leqq r<\infty}\left|\alpha_{1}(r, \varepsilon)-\alpha_{2}(r, \varepsilon)\right|, \sup _{1 \leqq r<\infty}\left|\beta_{1}(r, \varepsilon)-\beta_{2}(r, \varepsilon)\right|\right\}
$$

It is easy to show that $\rho_{\varepsilon}$ constitutes a metric on $C_{M}(\Omega)$, and that $C_{M}(\Omega)$ is a complete metric space. 
The right-hand sides of (3.5) and (3.6) can be regarded as a mapping defined on $C_{M}(\Omega)$, and we may write those equations in the form

$$
\tilde{\xi}=T(\xi),
$$

where the operator $T:(\alpha, \beta) \rightarrow(\tilde{\alpha}, \tilde{\beta})$ is given by

$$
\begin{aligned}
& \tilde{\alpha}(r, \varepsilon)=1+\varepsilon \int_{1}^{\infty} A(s, r, \varepsilon) \alpha(s, \varepsilon) \beta(s, \varepsilon) d s, \\
& \tilde{\beta}(r, \varepsilon)=1+\varepsilon \int_{1}^{\infty} B(s, r, \varepsilon) \alpha(s, \varepsilon) \beta(s, \varepsilon) d s .
\end{aligned}
$$

We can now prove the following.

LEMMA 5. The operator T maps the space $C_{M_{0}}(\Omega)$ into itself, where $M_{0}$ is any value of $M$ which satisfies the inequality

$$
1+h_{k} M^{2}-M \leqq 0
$$

for all $\varepsilon \in\left(0, \varepsilon_{0}\right]$.

Proof. When $\xi \in C_{M}(\Omega)$, equation (3.21) gives

$$
|\tilde{\alpha}| \leqq 1+M^{2} \varepsilon \int_{1}^{\infty}|A(s, r, \varepsilon)| d s \leqq 1+M^{2} h_{k}
$$

on application of Lemma 4. Hence the image $\tilde{\alpha}$ obeys $|\tilde{\alpha}| \leqq M$ when (3.23) holds. A similar result can be obtained for $\tilde{\beta}$, and hence the result follows.

LEMMA 6. The operator $T$ is a contraction on $C_{M_{0}}(\Omega)$ when

$$
M_{0}<1 /\left(2 h_{k}\right) .
$$

Proof. Let $\xi_{1}, \xi_{2}$ be arbitrary elements of $C_{M_{0}}(\Omega)$, whose images under $T$ are $\tilde{\xi}_{1}, \tilde{\xi}_{2}$ respectively. Then (3.21) gives

$$
\left|\tilde{\alpha}_{1}-\tilde{\alpha}_{2}\right| \leqq \varepsilon \cdot \sup _{r}\left|\alpha_{1} \beta_{1}-\alpha_{2} \beta_{2}\right| \cdot \int_{1}^{\infty}|A(s, r, \varepsilon)| d s .
$$

Noting that

$$
\left|\alpha_{1} \beta_{1}-\alpha_{2} \beta_{2}\right| \leqq M_{0}\left|\alpha_{1}-\alpha_{2}\right|+M_{0}\left|\beta_{1}-\beta_{2}\right|
$$

and using Lemma 4, we can write (3.25) as

$$
\left|\tilde{\alpha}_{1}-\tilde{\alpha}_{2}\right| \leqq h_{k} \cdot 2 M_{0} \rho_{\varepsilon}\left(\xi_{1}, \xi_{2}\right) \text {. }
$$

Similarly (3.22) gives

$$
\left|\tilde{\beta}_{1}-\tilde{\beta}_{2}\right| \leqq h_{k} \cdot 2 M_{0} \rho_{\varepsilon}\left(\xi_{1}, \xi_{2}\right) .
$$

Combining (3.26) and (3.27) we obtain

$$
\rho_{\varepsilon}\left(\tilde{\xi}_{1}, \tilde{\xi}_{2}\right) \leqq 2 h_{k} M_{0} \rho_{\varepsilon}\left(\xi_{1}, \xi_{2}\right)
$$

which shows that $T$ is a contraction when (3.24) holds, that is, when

$$
\lambda \equiv 2 h_{k} M_{0}<1 .
$$

The contraction mapping theorem ([6, p. 27]) now gives the following. 
Lemma 7. The system (3.21)-(3.22) has a unique fixed point on the space $C_{M_{0}}$, where $M_{0}$ satisfies (3.23) and (3.24).

Since $M_{0}$ can be chosen arbitrarily, provided that the inequalities (3.23) and (3.24) are satisfied, we have as an immediate consequence of Lemma 7 the following.

THEOREM 1. The integral equations (3.5) and (3.6) possess a unique continuous solution obeying the conditions (2.31).

Lemmas 2 and 3, which established a certain equivalence between equations (3.5) and (3.6) on the one hand and Problem A on the other hand, now give the main result.

THEOREM 2. For sufficiently small $\varepsilon>0$ Problem A has a unique solution, and this solution is representable in the form (2.16).

4. Asymptotic expansions. It is a consequence of the contraction mapping theorem that the fixed point of the operator $T$ is the limit of an iteration procedure which can begin with an arbitrary initial element $\xi_{0} \in C_{M_{0}}(\Omega)$. Moreover, if $\xi$ denotes the fixed point of $T$ and if

$$
\xi_{n}=T\left(\xi_{n-1}\right), \quad n=1,2, \cdots,
$$

then we have $([6$, p. 28$])$ the standard error estimate

$$
\rho_{\varepsilon}\left(\xi, \xi_{n}\right) \leqq \frac{\lambda^{n}}{1-\lambda} \rho_{\varepsilon}\left(\xi_{0}, \xi_{1}\right), \quad n \geqq 1,
$$

where $\lambda<1$ is the contraction parameter (3.29).

Let the initial element $\xi_{0}=\left(\alpha_{0}, \beta_{0}\right)$ be

$$
\alpha_{0}(r, \varepsilon) \equiv 0, \quad \beta_{0}(r, \varepsilon) \equiv 0 .
$$

Then it follows directly from (3.21), (3.22) and (4.1) that the first iterate $\xi_{1}=\left(\alpha_{1}, \beta_{1}\right)$ is

$$
\alpha_{1}(r, \varepsilon)=1, \quad \beta_{1}(r, \varepsilon)=1
$$

Evidently

$$
\rho_{\varepsilon}\left(\xi_{0}, \xi_{1}\right)=1
$$

and hence

$$
\begin{aligned}
\rho_{\varepsilon}\left(\xi, \xi_{1}\right) & =\max \left\{\sup _{r}|\alpha(r, \varepsilon)-1|, \sup _{r}|\beta(r, \varepsilon)-1|\right\} \\
& \leqq \frac{\lambda}{1-\lambda} \rho_{\varepsilon}\left(\xi_{0}, \xi_{1}\right) \leqq \frac{\lambda}{1-\lambda} .
\end{aligned}
$$

Since $\lambda=O\left(h_{k}\right)$, where $h_{k}$ is given by (3.11), we thus have

$$
\lim _{\varepsilon \rightarrow 0} \rho_{\varepsilon}\left(\xi, \xi_{1}\right)=0
$$

and

$$
\alpha(r, \varepsilon)=1+O\left(h_{k}\right), \quad \beta(r, \varepsilon)=1+O\left(h_{k}\right)
$$

as $\varepsilon \rightarrow 0$, uniformly on $1 \leqq r<\infty$. 
Referring to the definitions (2.9) and (2.16) we see that we have proved the following theorem.

THEOREM 3. The solution of Problem $\mathrm{B}$ is an asymptotic approximation as $\varepsilon \rightarrow 0$ to the solution of Problem A, with

$$
u(r, \varepsilon) \sim U(r, \varepsilon), \quad u^{\prime}(r, \varepsilon) \sim U^{\prime}(r, \varepsilon)
$$

uniformly on $1 \leqq r<\infty$.

We turn our attention now to the $n$th iterate derived from (4.1). First we have from (4.2) and (4.5) that

$$
\rho_{\varepsilon}\left(\xi, \xi_{n}\right) \leqq \lambda^{n} /(1-\lambda)
$$

which implies that

$$
\begin{aligned}
& \alpha(r, \varepsilon)=\alpha_{n}(r, \varepsilon)+O\left[h_{k}^{n}\right], \\
& \beta(r, \varepsilon)=\beta_{n}(r, \varepsilon)+O\left[h_{k}^{n}\right] .
\end{aligned}
$$

Next, it follows from the triangle inequality that

by (4.10). Hence

$$
\begin{aligned}
\rho_{\varepsilon}\left(\xi_{n-1}, \xi_{n}\right) & \leqq \rho_{\varepsilon}\left(\xi, \xi_{n-1}\right)+\rho_{\varepsilon}\left(\xi, \xi_{n}\right) \\
& \leqq \frac{\lambda^{n-1}}{1-\lambda}+\frac{\lambda^{n}}{1-\lambda},
\end{aligned}
$$

$$
\rho_{\varepsilon}\left(\xi_{n-1}, \xi_{n}\right) \leqq C_{k, n} h_{k}^{n-1},
$$

where $C_{k, n}=\left(\left(1+2 M_{0} h_{k}\right) /\left(1-2 M_{0} h_{k}\right)\right)\left(2 M_{0}\right)^{n-1}=O(1)$ as $\varepsilon \rightarrow 0$. From (4.12) we infer that

$$
\begin{aligned}
& \alpha_{n}(r, \varepsilon)=\alpha_{n-1}(r, \varepsilon)+h_{k}^{n-1} \phi_{n-1}(r, \varepsilon) \\
& \beta_{n}(r, \varepsilon)=\beta_{n-1}(r, \varepsilon)+h_{k}^{n-1} \psi_{n-1}(r, \varepsilon)
\end{aligned}
$$

where the functions $\phi_{n-1}$ and $\psi_{n-1}$ are uniformly $O(1)$ on $1 \leqq r<\infty, 0<\varepsilon \leqq \varepsilon_{0}$.

Combining (4.11) and (4.13) we obtain the asymptotic expansions

$$
\begin{aligned}
& \alpha(r, \varepsilon)=\sum_{n=0}^{N-1} h_{k}^{n} \phi_{n}(r, \varepsilon)+O\left(h_{k}^{N}\right), \\
& \beta(r, \varepsilon)=\sum_{n=0}^{N-1} h_{k}^{n} \psi_{n}(r, \varepsilon)+O\left(h_{k}^{N}\right)
\end{aligned}
$$

as $\varepsilon \rightarrow 0$. Note that, because of (4.8), $\phi_{0}=\psi_{0}=1$.

Substituting (4.14) into (2.9) and (2.16) we achieve asymptotic expansions for $u(r, \varepsilon)$ and $u^{\prime}(r, \varepsilon)$. In particular we have the following.

THEOREM 4. The solution to Problem A has the generalized asymptotic expansion

$$
u(r, \varepsilon)=U(r, \varepsilon)+\sum_{n=1}^{N} v_{n}(\varepsilon) u_{n}(r, \varepsilon)+O\left[v_{N+1}(\varepsilon)\right]
$$

where

$$
v_{n}(\varepsilon)=h_{k}^{n}(\varepsilon)
$$


and

$$
u_{n}(r, \varepsilon)=-W(r, \varepsilon) \phi_{n}(r, \varepsilon) .
$$

In conclusion it may be noted that Theorem 4 provides an algorithm for calculating a uniformly valid asymptotic approximation to $u(r, \varepsilon)$ correct to any order. From a practical point of view it is probably easiest to perform the calculation by writing $u(r, \varepsilon)$ in the form

$$
u(r, \varepsilon)=1-\alpha(r, \varepsilon) W(r, \varepsilon)
$$

and applying the iteration formulas (4.1), (4.11) and (4.14) to obtain the required approximation to $u(r, \varepsilon)$. Thus, for example, the second approximation is

$$
u(r, \varepsilon)=1-\alpha_{2}(r, \varepsilon) W(r, \varepsilon)+O\left(h_{k}^{2}\right)
$$

where

$$
\begin{aligned}
\alpha_{2}(r, \varepsilon) & =T\left(\alpha_{1}(r, \varepsilon)\right)=T(1) \\
& =1+\varepsilon \int_{1}^{\infty} A(s, r, \varepsilon) d s .
\end{aligned}
$$

The question of whether this method is applicable to more general problems is under investigation.

\section{REFERENCES}

[1] M. Abramowitz and I. A. Stegun, Handbook of Mathematical Functions, National Bureau of Standards, Washington D.C., 1965.

[2] W. B. Bush, On the Lagerstrom mathematical model for viscous flow at low Reynolds number, this Journal, 20 (1971), pp. 279-287.

[3] J. D. Cole, Perturbation Methods in Applied Mathematics, Blaisdell, New York, 1968.

[4] G. C. HsiaO, Singular perturbations for a nonlinear differential equation with a small parameter, SIAM J. Math. Anal., 4 (1973), pp. 283-301.

[5] P. A. Lagerstrom, Asymptotic methods for the study of the Navier-Stokes equations, Calif. Instit. Tech. Translation Project, Pasadena, Calif., 1965; see also P. A. Lagerstrom And R. G. CASTEN, Basic concepts underlying singular perturbation techniques, SIAM Rev., 14 (1972), pp. 63-120.

[6] L. LiUSTERniK AND V. Sobolev, Elements of Functional Analysis, Ungar, New York, 1965.

[7] J. G. VAN DER Corput, Asymptotic developments, I. Fundamental theorems of asymptotics, J. Analyse Math., 4 (1955), pp. 341-418. 